\title{
Simulation numérique d'un tube flexible soumis à un écoulement interne
}

\author{
Fabien Huvelin ${ }^{1, a}$, Élisabeth Longatte ${ }^{1}$ et Mhamed Souli $^{2}$ \\ 1 EDF R\&D, Département Mécanique des Fluides, Énergies et Environnement, 6 quai Watier, 78400 Chatou, France \\ 2 Laboratoire de Mécanique de Lille, Université des Sciences et Technologies de Lille 1, Cité Scientifique, \\ 1 boulevard Paul Langevin, 59655 Lille, France
}

Reçu le $1^{\text {er }}$ septembre 2006, accepté le 23 janvier 2007

\begin{abstract}
Résumé - Cet article est consacré à la simulation numérique, par couplage externe de codes, de vibrations de structures flexibles induites par écoulements. Une revue des développements réalisés sur les deux codes disciplinaires mis en œuvre est tout d'abord présentée, puis une application au cas d'un tube flexible soumis à un écoulement interne pour de petits déplacements et un écoulement laminaire est proposée.
\end{abstract}

Mots clés : Interaction fluide-structure / couplage de codes externes / départ en instabilité / formulation ALE / structure flexible

\begin{abstract}
Numerical simulation of a pipe conveying fluid. The present work is devoted to numerical simulation of flow induced vibrations by means of a partitioned code coupling procedure. First, fluid and structure codes are presented. Then the computation of a pipe conveying fluid for small displacement and laminar flow is investigated.
\end{abstract}

Key words: Fluid-structure interaction / partitioned algorithm / instability departure / ALE formulation / flexible structure

\section{Introduction}

Les méthodes numériques pour les calculs d'écoulements monophasiques et de vibrations de structures sont arrivées à un point de maturité tel qu'il est désormais possible d'envisager des simulations couplées de ces phénomènes physiques. Pour cela, deux voies sont envisageables.

La première consiste à résoudre les équations fluide et structure dans un même système d'équations. Il s'agit d'algorithmes dits monolithiques [1-3] avec lesquels les propriétés de conservation sont assurées à l'interface permettant ainsi une stabilité inconditionnelle du schéma. Le choix du pas de temps est alors limité uniquement par la précision demandée. Mais ce type d'algorithme demande le développement complet d'un code de calcul. De plus, les méthodes numériques employées pour le fluide et la structure sont souvent différentes et il est difficile de les

\footnotetext{
a Auteur correspondant : fabien.huvelin@edf.fr
}

faire cohabiter au sein d'un même solveur, d'où l'utilisation répandue des algorithmes partitionnés.

Cette seconde méthode utilise des codes monodisciplinaires. La démarche consiste à mettre à jour les codes disciplinaires basés sur des formulations classiques, tels que les volumes finis dans un référentiel fixe (ou eulérien) pour le fluide et les éléments-finis dans un référentiel mobile (ou lagrangien) pour la structure, afin de prendre en considération les couplages entre les phénomènes physiques à l'interface des deux milieux. Pour cela, les équations sont résolues consécutivement en transférant les conditions à l'interface d'un solveur à l'autre. L'avantage est que l'on peut utiliser les codes existants et tirer parti des formulations optimisées pour chaque problème. L'inconvénient de ce type de résolution est que cela provoque un décalage en temps dans la résolution des problèmes. Pour résoudre le problème fluide entre les instants $n$ et $n+1$, il est nécessaire de savoir comment va se déplacer la structure entre ces deux temps. Inversement, pour résoudre le problème structure entre les instants $n$ et $n+1$, il est nécessaire de connaître les efforts appliqués par le 


\section{Nomenclature}

\begin{tabular}{|c|c|c|}
\hline$A$ & Amplitude initiale de la structure & $\left(\mathrm{m} \cdot \mathrm{s}^{-1}\right)$ \\
\hline$\overline{\bar{D}}_{\mathrm{f}}$ & Tenseur des vitesses de déformations & $\left(\mathrm{s}^{-1}\right)$ \\
\hline$e$ & Épaisseur du tube & $(\mathrm{m})$ \\
\hline$E_{\mathrm{s}}$ & Module d'Young & $\left(\mathrm{kg} \cdot \mathrm{m} \cdot \mathrm{s}^{-2}\right)$ \\
\hline$f_{\mathrm{f}}$ & Forces extérieures appliquées au fluide & $\left(\mathrm{kg} \cdot \mathrm{m} \cdot \mathrm{s}^{-2}\right)$ \\
\hline$f_{\mathrm{s}}$ & Forces extérieures appliquées à la structure & $\left(\mathrm{kg} \cdot \mathrm{m} \cdot \mathrm{s}^{-2}\right)$ \\
\hline $\boldsymbol{F}_{\mathrm{s}}^{\mathrm{n}}$ & Efforts appliqués sur la structure à l'interface fluide-structure & $\left(\mathrm{kg} \cdot \mathrm{m} \cdot \mathrm{s}^{-2}\right)$ \\
\hline$\underline{F}_{\mathrm{f}}^{\mathrm{n}}$ & Efforts appliqués par le fluide sur l'interface fluide-structure & $\left(\mathrm{kg} \cdot \mathrm{m} \cdot \mathrm{s}^{-2}\right)$ \\
\hline$\overline{\bar{I}}$ & Tenseur identité & $(-)$ \\
\hline$J$ & Jacobien entre les référentiels eulérien et ALE & $(-)$ \\
\hline$L$ & Longueur du tube & $(\mathrm{m})$ \\
\hline$p$ & Pression & $\left(\mathrm{kg} \cdot \mathrm{m}^{-2} \cdot \mathrm{s}^{-2}\right)$ \\
\hline$R_{\mathrm{i}}$ & Rayon interne du tube & $(\mathrm{m})$ \\
\hline$t$ & Temps & $(\mathrm{s})$ \\
\hline $\boldsymbol{u}_{\mathrm{s}}$ & Déplacement de la structure & $(\mathrm{m})$ \\
\hline$\dot{u}_{\mathrm{s}}$ & Vitesse de la structure & $\left(\mathrm{m} \cdot \mathrm{s}^{-1}\right)$ \\
\hline $\boldsymbol{v}_{\text {ale }}$ & Vitesse de maillage & $\left(\mathrm{m} . \mathrm{s}^{-1}\right)$ \\
\hline $\boldsymbol{v}_{\mathrm{f}}$ & Vitesse du fluide & $\left(\mathrm{m} . \mathrm{s}^{-1}\right)$ \\
\hline $\boldsymbol{u}_{\mathrm{ifs}}$ & Déplacement de l'interface fluide-structure & $(\mathrm{m})$ \\
\hline $\bar{v}_{\text {red }}$ & Vitesse réduite adimensionnée & $(-)$ \\
\hline$x$ & Coordonnées dans le référentiel eulérien & $(\mathrm{m})$ \\
\hline$\alpha_{0}$ & Constante du schéma de couplage explicite synchrone & $(-)$ \\
\hline$\alpha_{1}$ & Constante du schéma de couplage explicite synchrone & $(-)$ \\
\hline$\Delta t$ & Pas de temps & $(\mathrm{s})$ \\
\hline$\overline{\bar{\epsilon}}$ & Tenseur des déformations & $(-)$ \\
\hline$\Gamma_{\mathrm{f}}^{n}$ & Interface fluide au temps $n$ & $(-)$ \\
\hline$\Gamma_{\mathrm{s}}^{n}$ & Interface structure au temps $n$ & $(-)$ \\
\hline$\Gamma_{\mathrm{s} / \mathrm{f}}^{n}$ & Interface fluide-structure au temps $n$ & $(-)$ \\
\hline$\lambda_{1}$ & Coefficient de Lamé & $\left(\mathrm{kg} \cdot \mathrm{m}^{-1} \cdot \mathrm{s}^{-2}\right)$ \\
\hline$\mu$ & Viscosité dynamique & $\left(\mathrm{kg} \cdot \mathrm{m}^{-1} \cdot \mathrm{s}^{-1}\right)$ \\
\hline$\mu_{1}$ & Coefficient de Lamé & $\left(\mathrm{kg} \cdot \mathrm{m}^{-1} \cdot \mathrm{s}^{-2}\right)$ \\
\hline$\nu_{\mathrm{s}}$ & Coefficient de Poisson & $(-)$ \\
\hline$\Omega^{\mathrm{n}}$ & Domaine de calcul au temps $n$ & $(-)$ \\
\hline$\Omega_{\mathrm{f}}^{\mathrm{n}}$ & Domaine fluide au temps $n$ & $(-)$ \\
\hline$\Omega_{\mathrm{s}}^{\mathrm{n}}$ & Domaine structure au temps $n$ & $(-)$ \\
\hline$\rho_{\mathrm{f}}$ & Masse volumique fluide & $\left(\mathrm{kg} \cdot \mathrm{m}^{-3}\right)$ \\
\hline$\rho_{\mathrm{s}}$ & Masse volumique structure & $\left(\mathrm{kg} \cdot \mathrm{m}^{-3}\right)$ \\
\hline$\overline{\bar{\sigma}}_{\mathrm{s}}$ & Tenseur des contraintes structures & $\left(\mathrm{kg} \cdot \mathrm{s}^{-2}\right)$ \\
\hline$\overline{\bar{\sigma}}_{\mathrm{f}}$ & Tenseur des contraintes fluides & $\left(\mathrm{kg} \cdot \mathrm{s}^{-2}\right)$ \\
\hline$\theta$ & Trace du tenseur des déformations & $(-)$ \\
\hline$\xi$ & Coordonnées dans le référentiel ALE & $(\mathrm{m})$ \\
\hline
\end{tabular}

fluide sur la structure pendant cette période. Afin de pallier à ce problème, des schémas de couplage optimisés ont été mis en œuvre [4-6]. Les données transférées entre les deux codes sont les efforts du fluide vers la structure et les déplacements et les vitesses de la structure vers le fluide. Généralement les maillages des deux milieux ont des raffinements différents, il est donc nécessaire d'interpoler les données lors du transfert [7-9]. De plus, le maillage structure étant mobile, il est nécessaire d'adapter le code fluide afin de pouvoir correctement appliquer les déplacements structure sur le maillage fluide. Une possibilité est de modifier les équations fluide afin de pouvoir déformer la grille de calcul fluide grâce à une formulation Arbitraire Euler Lagrange (ALE) [10,11].

Un couplage fluide-structure par algorithme partitionné demande donc le développement des trois points suivants :

- la définition d'un schéma de couplage, ou prédicteur, entre les deux codes,

- le traitement des conditions aux limites structure à l'interface du problème fluide (par la mise en œuvre 
d'une déformation de maillage du domaine fluide, par exemple),

- l'interpolation des données fluide et structure à l'interface afin de transférer les données d'un code à l'autre.

\section{Méthodologie de couplage}

\subsection{Formulation du problème fluide-structure}

Considérons une structure qui, un instant $t=n$, occupe un domaine $\Omega_{\mathrm{s}}^{n} \subset \Omega^{\mathrm{n}}$ et un fluide occupant au même instant un domaine $\Omega_{\mathrm{f}}^{n}=\Omega^{\mathrm{n}}-\Omega_{\mathrm{s}}^{n}$. On définit au même instant les bords des domaines fluide et structure par, respectivement, $\Gamma_{\mathrm{f}}^{n}$ et $\Gamma_{\mathrm{s}}^{n}$ et l'interface entre les deux milieux $\operatorname{par} \Gamma_{\mathrm{f} / \mathrm{s}}^{n}=\Gamma_{\mathrm{s}}^{n} \cap \Gamma_{\mathrm{f}}^{n}$.

\section{Problème structure}

La formulation locale de l'équation de la quantité de mouvement dans le référentiel lagrangien s'écrit :

$$
\left\{\begin{aligned}
\rho_{\mathrm{s}} \frac{\mathrm{d}^{2} \boldsymbol{u}_{\mathrm{s}}}{\mathrm{d} t^{2}} & =\boldsymbol{f}_{\mathrm{s}}+\nabla \cdot\left(\overline{\overline{\boldsymbol{\sigma}}}_{\mathrm{s}}\right) & & \text { sur } \Omega_{\mathrm{s}} \\
\overline{\overline{\boldsymbol{\sigma}}}_{\mathrm{s}} \cdot \boldsymbol{n} & =\overline{\overline{\boldsymbol{\sigma}}}_{\mathrm{f}} \cdot \boldsymbol{n} & & \text { sur } \Gamma_{\mathrm{s}, \mathrm{f}} \\
\overline{\overline{\boldsymbol{\sigma}}}_{\mathrm{s}} \cdot \boldsymbol{n} & =0 & & \text { sur } \Gamma_{\mathrm{s}} / \Gamma_{\mathrm{s}, \mathrm{f}} \\
\boldsymbol{u}_{\mathrm{s}} & =\boldsymbol{u}_{\mathrm{f}} & & \text { sur } \Gamma_{\mathrm{s}, \mathrm{f}} \\
\boldsymbol{u}_{\mathrm{s}} & =0 & & \text { sur } \Gamma_{\mathrm{s}} / \Gamma_{\mathrm{s}, \mathrm{f}}
\end{aligned}\right.
$$

À cette équation s'ajoute une loi de comportement du matériau étudié. Dans le domaine élastique linéaire, le tenseur des contraintes est défini par la loi de Lamé :

$$
\overline{\overline{\boldsymbol{\sigma}}}_{\mathrm{s}}=\lambda_{\mathrm{l}} \theta \overline{\overline{\boldsymbol{I}}}+2 \mu_{\mathrm{l}} \overline{\bar{\varepsilon}}_{\mathrm{s}}
$$

avec $\overline{\bar{\varepsilon}}_{\mathrm{s}}=\frac{1}{2}\left(\overline{\overline{\boldsymbol{\nabla} \boldsymbol{u}_{\mathrm{s}}}}+{ }^{\mathrm{t}} \overline{\overline{\nabla \boldsymbol{u}_{\mathrm{s}}}}\right)$ le tenseur de déformation, $\theta$ la trace de $\overline{\bar{\varepsilon}}, \overline{\bar{I}}$ le tenseur unité et $\lambda_{\mathrm{l}}$ et $\mu_{\mathrm{l}}$ les coefficients de Lamé.

\section{Problème fluide et formulation ALE}

Le fluide est considéré incompressible. La formulation locale des équations de conservation de la masse et de la quantité de mouvement dans le référentiel eulérien s'écrit :

$$
\left\{\begin{aligned}
\nabla \cdot\left(\boldsymbol{v}_{\mathrm{f}}\right) & =0 & & \operatorname{sur} \Omega_{\mathrm{f}} \\
\frac{\partial \rho_{\mathrm{f}} \boldsymbol{v}_{\mathrm{f}}}{\partial t}+\nabla \cdot\left(\rho_{\mathrm{f}} \boldsymbol{v}_{\mathrm{f}} \otimes \boldsymbol{v}_{\mathrm{f}}\right) & =\boldsymbol{f}_{\mathrm{f}}+\nabla \cdot\left(\overline{\overline{\boldsymbol{\sigma}}}_{\mathrm{f}}\right) & & \text { sur } \Omega_{\mathrm{f}} \\
\overline{\overline{\boldsymbol{\sigma}}}_{\mathrm{f}} \cdot \boldsymbol{n} & =\overline{\overline{\boldsymbol{\sigma}}}_{\mathrm{s}} \cdot \boldsymbol{n} & & \text { sur } \Gamma_{\mathrm{s}, \mathrm{f}} \\
\overline{\overline{\boldsymbol{\sigma}}}_{\mathrm{f}} \cdot \boldsymbol{n} & =0 & & \text { sur } \Gamma_{\mathrm{f}} / \Gamma_{\mathrm{s}, \mathrm{f}} \\
\boldsymbol{u}_{\mathrm{f}} & =\boldsymbol{u}_{\mathrm{s}} & & \text { sur } \Gamma_{\mathrm{s}, \mathrm{f}} \\
\boldsymbol{u}_{\mathrm{f}} & =0 & & \text { sur } \Gamma_{\mathrm{f}} / \Gamma_{\mathrm{s}, \mathrm{f}}
\end{aligned}\right.
$$
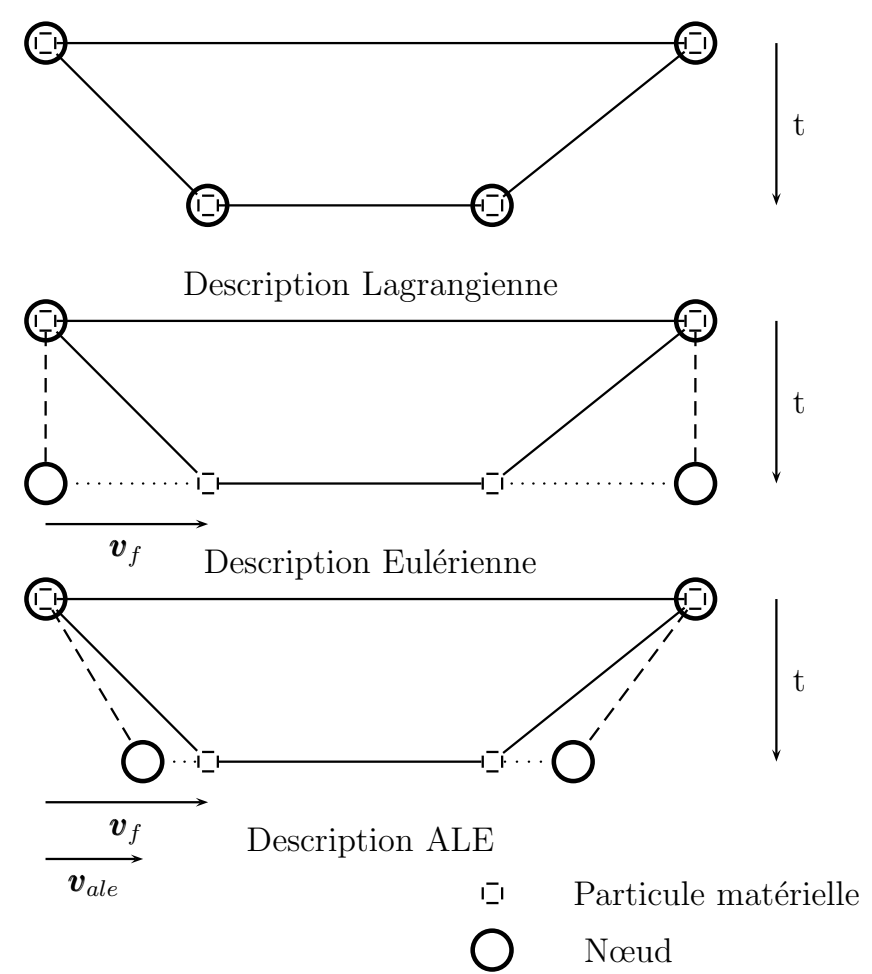

Fig. 1. Référentiel lagrangien, eulérien et ALE.

À ces équations s'ajoute une loi de comportement du fluide modélisé. Pour un fluide newtonien en écoulement laminaire, le tenseur des contraintes s'écrit :

$$
\overline{\overline{\boldsymbol{\sigma}}}_{\mathrm{f}}=-p \overline{\overline{\boldsymbol{I}}}+2 \mu \overline{\overline{\boldsymbol{D}}}_{\mathrm{f}}
$$

avec $\overline{\overline{\boldsymbol{D}}}_{\mathrm{f}}=\frac{1}{2}\left(\overline{\overline{\boldsymbol{\nabla} \boldsymbol{v}_{\mathrm{f}}}}+{ }^{\mathrm{t}} \overline{\overline{\boldsymbol{\nabla} \boldsymbol{v}_{\mathrm{f}}}}\right)$ le tenseur des vitesses de déformation.

Afin de suivre l'interface structure, la méthode Arbitraire Lagrange-Euler est utilisée. Elle consiste à définir un référentiel arbitraire dans lequel vont être calculées les variables physiques fluide. Dans ce référentiel, les nœuds du maillage se déplacent d'une valeur arbitraire comprise entre celles des descriptions lagrangienne et eulérienne (Fig. 1).

Il apparait une vitesse arbitraire, dite vitesse de maillage $\boldsymbol{v}_{\text {ale }}$, qui permet de se rapprocher d'une formulation lagrangienne lorsque cette vitesse est égale à la vitesse du fluide, ce qui est intéressant lorsque l'on est proche de l'interface fluide-structure afin de pouvoir suivre la description lagrangienne de la structure, et de se rapprocher d'une formulation eulérienne lorsque cette vitesse s'approche de zéro, ce qui est intéressant loin de l'interface fluide-structure où le maillage n'a pas besoin d'être déplacé. Le calcul dans un tel domaine implique une reformulation des équations du fluide dans ce repère. La relation fondamentale entre le référentiel eulérien et le référentiel ALE est :

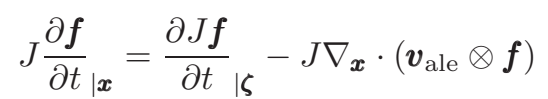


En appliquant la formule (5) à (3), on obtient une formulation ALE des équations de conservation de la masse et de la quantité de mouvement pour le fluide :

$$
\left\{\begin{aligned}
\nabla_{\boldsymbol{x}} \cdot\left(\boldsymbol{v}_{\mathrm{f}}-\boldsymbol{v}_{\text {ale }}\right) & =0 \\
\frac{\partial J \rho_{\mathrm{f}} \boldsymbol{v}_{\mathrm{f}}}{\partial t}+J\left(\nabla_{\boldsymbol{x}} \cdot\left(\rho_{\mathrm{f}}\left(\boldsymbol{v}_{\mathrm{f}}-\boldsymbol{v}_{\text {ale }}\right) \otimes \boldsymbol{v}_{\mathrm{f}}\right)\right) & = \\
J\left(\boldsymbol{f}_{\mathrm{f}}+\nabla_{\boldsymbol{x}} \cdot\left(\overline{\bar{\sigma}}_{\mathrm{f}}\right)\right) &
\end{aligned}\right.
$$

Lorsque la discrétisation est effectuée à l'ordre 1, les simplifications suivantes sont possibles : $J=I d, \nabla_{\boldsymbol{\zeta}}=\nabla_{\boldsymbol{x}}$ et $\boldsymbol{v}_{\mathrm{f}}^{\mathrm{a}}(\boldsymbol{\zeta}, t)=\boldsymbol{v}_{\mathrm{f}}^{\mathrm{e}}(\boldsymbol{x}, t)$. De plus, [12] a montré que pour un fluide incompressible on peut négliger la vitesse de maillage $\boldsymbol{v}_{\text {ale }}$ dans l'équation de conservation de la masse.

\subsection{Schéma de couplage}

La seconde difficulté à surmonter dans l'utilisation d'un algorithme partionné est la résolution consécutive des deux problèmes. Supposons qu'à un instant $t^{n+1}$ le déplacement de l'interface soit donné $\boldsymbol{u}_{\mathrm{ifs}}^{n+1}$. Il est alors possible de résoudre les équations fluides entre un instant $t^{n}$ et $t^{n+1}$ et d'en déduire les efforts qui s'appliquent à la paroi fluide-structure : $\boldsymbol{F}_{\mathrm{f}}^{n+1}=\mathcal{F}\left(\boldsymbol{u}_{\mathrm{ifs}}^{n+1}\right)$. Supposons, maintenant, que les efforts exercés par le fluide sur l'interface sont donnés au temps $t^{n+1}$. Il est alors possible de calculer le déplacement de la structure et donc de l'interface entre les instants $t^{n}$ et $t^{n+1}: \boldsymbol{u}_{\mathrm{ifs}}^{n+1}=\mathcal{U}\left(\boldsymbol{F}_{\mathrm{f}}^{n+1}\right)$. Le problème du déplacement de l'interface fluide-structure peut alors s'écrire sous la forme : $\boldsymbol{u}_{\mathrm{ifs}}^{n+1}=\mathcal{U} \circ \mathcal{F}\left(\boldsymbol{u}_{\mathrm{ifs}}^{n+1}\right)$. Afin de pouvoir résoudre ce problème, deux types de résolution sont envisagés : une méthode explicite et une méthode implicite. Afin de minimiser au mieux la création d'énergie à l'interface, des schémas explicites et des schémas par sous-cyclages ont été proposés. Une comparaison des performances des schémas présentés ci-après pourra être trouvée dans [13].

\section{Algorithme explicite}

Le principe de l'algorithme explicite est de prédire la position du domaine de calcul à l'instant $t^{n+1}$ en fonction des variables calculées aux temps $t^{n}$ et $t^{n-1}$ :

$$
\boldsymbol{u}_{\mathrm{ifs}}^{n+1}=\boldsymbol{u}_{\mathrm{s}}^{n}+\alpha_{0} \Delta t \dot{\boldsymbol{u}}_{\mathrm{s}}^{n}+\alpha_{1} \Delta t\left(\dot{\boldsymbol{u}}_{\mathrm{s}}^{n}-\dot{\boldsymbol{u}}_{\mathrm{s}}^{n-1}\right)
$$

où $\alpha_{0}$ et $\alpha_{1}$ sont des constantes. Le schéma de couplage est du second ordre si l'on choisit $\alpha_{0}=1$ et $\alpha_{1}=0,5$ et si l'on assure que les forces que l'on applique sur la structure $\boldsymbol{F}_{\mathrm{s}}$ sont interpolées en fonction des efforts calculés sur l'interface fluide $\boldsymbol{F}_{\mathrm{f}}$ de la manière suivante :

$$
\boldsymbol{F}_{\mathrm{f}}^{n}=\frac{\boldsymbol{F}_{\mathrm{s}}^{n+1}+\boldsymbol{F}_{\mathrm{s}}^{n}}{2}
$$

Ce schéma de couplage a été proposé par [14] et utilisé avec succès pour des problèmes d'aéroélasticité [15]. Nous pouvons citer le cas particulier où $\alpha_{0}=\frac{1}{2}$ et $\alpha_{1}=0$, dit schéma asynchrone, qui prédit la position du domaine de calcul et résoud le problème fluide à l'instant $t^{n+1 / 2}$ en fonction des variables structures calculées au temps $t^{n}$. Cette prédiction a l'avantage de conserver la géométrie sans violer la continuité des champs de vitesses à l'interface entre les deux milieux [16].

\section{Algorithme par sous-cyclages}

Le principe de l'algorithme par sous-cyclages consiste à sous-itérer un pas de temps jusqu'à ce que la précision de la prédiction de l'interface soit jugée suffisante. Soient un état fluide et un état structure donnés au temps $t^{n}$. Les sous-itérations $k$ du pas de temps $n+1$ sont effectuées de la manière suivante :

- Prédiction du déplacement de l'interface

$$
\boldsymbol{u}_{\mathrm{ifs}}^{n+1, k}=\boldsymbol{u}_{\mathrm{ifs}}\left(\boldsymbol{u}_{\mathrm{s}}^{n+1, k-1}, \dot{\boldsymbol{u}}_{\mathrm{s}}^{n+1, k-1}\right)
$$

- Résolution des équations fluide

$$
\begin{aligned}
& p^{n+1, k}=p\left(p^{n}, \boldsymbol{v}_{\mathrm{f}}^{n}, \boldsymbol{u}_{\mathrm{ifs}}^{n+1, k}\right) \\
& \boldsymbol{v}_{\mathrm{f}}^{n+1, k}=\boldsymbol{v}\left(p^{n}, \boldsymbol{v}_{\mathrm{f}}^{n}, \boldsymbol{u}_{\mathrm{ifs}}^{n+1, k}\right)
\end{aligned}
$$

- Calcul des efforts fluide à appliquer à la structure

$$
\boldsymbol{F}_{\mathrm{f}}^{n+1, k}=\boldsymbol{F}\left(p^{n+1, k}, \boldsymbol{v}_{\mathrm{f}}^{n+1, k}\right)
$$

- Résolution des équations structure

$$
\boldsymbol{u}_{\mathrm{s}}^{n+1, k}=\boldsymbol{u}\left(\boldsymbol{u}_{\mathrm{s}}^{n}, \dot{\boldsymbol{u}}_{\mathrm{s}}^{n}, \ddot{\boldsymbol{u}}_{\mathrm{s}}^{n}, \boldsymbol{F}_{\mathrm{f}}^{n+1, k}\right)
$$

- Test de convergence sur le déplacement

$$
\left\|\frac{\boldsymbol{u}_{\mathrm{s}}^{n+1, k}-\boldsymbol{u}_{\mathrm{s}}^{n+1, k-1}}{\boldsymbol{u}_{\mathrm{s}}^{n+1,0}}\right\| \leq \varepsilon
$$

- Passage au prochain pas de temps ou à la prochaine sous-itération.

Cet algorithme de point fixe converge si la première estimation est proche de la solution recherchée. [6] propose une méthode de Newton-Raphson sur le calcul du déplacement de structures rigides afin d'améliorer la vitesse de convergence de l'algorithme.

\section{Transfert de données}

Lors de la simulation d'un phénomène en interaction fluide structure par un algorithme partitionné entre un code de mécanique des fluides et un code de mécanique des structures, un transfert de données doit être effectué à l'interface commune aux deux milieux :

- les efforts exercés par le fluide sur la structure, calculés aux points de discrétisation de l'interface du milieu fluide, doivent être transmis aux points de discrétisation de l'interface structure, 
MAILLAGE STRUCTURE

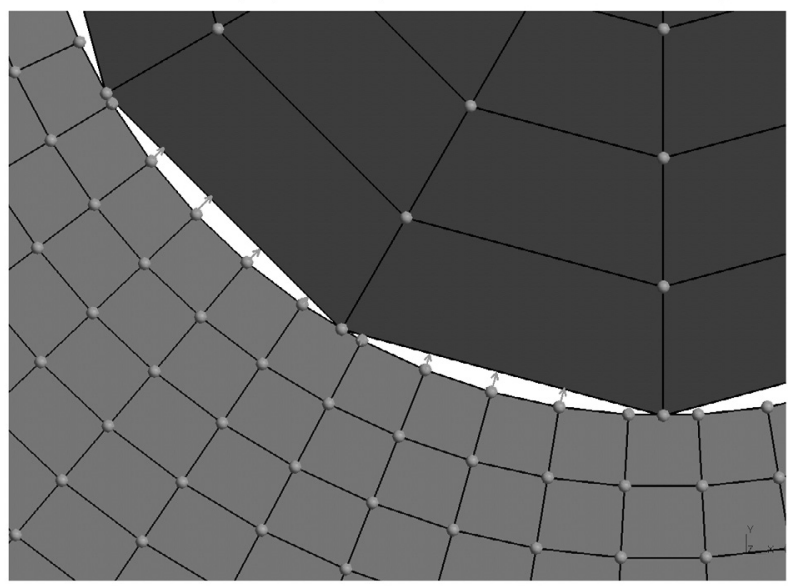

MAILLAGE FLUIDE

Fig. 2. Maillages des interfaces fluide et structures non conformes.

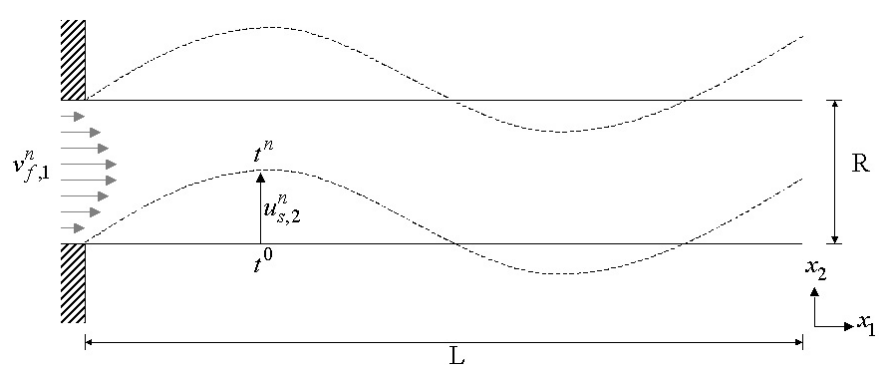

Fig. 3. Description du modèle.

- les déplacements de la structure, calculés aux points de discrétisation de son interface, doivent être transmis aux points de discrétisation de l'interface fluide.

On entend par points de discrétisation, les entités géométriques où est résolu chaque solveur. Typiquement, le modèle structure est formulé en éléments-finis et est résolu aux nœuds du maillage, tandis que le modèle fluide est formulé en volumes finis et est résolu aux centres des mailles. [8] et [9] proposent une méthode de projection basée sur les méthodes d'interpolation, assurant l'équilibre entre les efforts calculés sur l'interface du milieu fluide et ceux calculés sur l'interface du milieu structure. Il est supposé qu'il n'y a pas de condition de glissement entre les deux maillages. Chacun des points de discrétisation du milieu le plus raffiné peut donc être lié à un élément du maillage de l'autre milieu et ainsi être repéré durant l'ensemble de la simulation. Grâce à cette table de connectivité et à l'aide des fonctions d'interpolation (telles que les fonctions de forme), les données peuvent être transmises entre les deux maillages. Si l'on suppose le maillage fluide plus raffiné que le maillage structure (Fig. 2), alors :

- grâce à une fonction d'interpolation, la force définie en un point de discrétisation fluide est répartie sur les points de discrétisation de l'élément structure qui lui est associé,
- grâce à une fonction d'interpolation, le déplacement au point de discrétisation fluide est calculé en fonction des points de discrétisation de l'élément structure qui lui est associé.

\section{Application}

Afin de tester la procédure de couplage pour une structure flexible, le cas d'un tuyau soumis à un écoulement laminaire interne et des petits déplacements a été choisi (Fig. 3). L'intérêt est de mettre en évidence l'apparition d'une instabilité dynamique à partir d'une certaine vitesse d'écoulement. Des modèles analytiques ont été développés afin de comprendre les raisons de cette instabilité $[17,18]$. Pour un tube encastré en amont et libre en aval de l'écoulement, il a été démontré que le départ en instabilité porte sur le deuxième mode propre de la structure qui voit son amortissement devenir négatif à partir d'une certaine vitesse d'écoulement. Des comparaisons à des simulations numériques sont possibles. On peut citer celles de $[19,20]$ qui se basent sur des méthodes de transpiration.

\subsection{Modélisation}

La modélisation effectuée est 2D, puisque le modèle analytique ne présuppose pas une géométrie axisymétrique de la structure. L'écoulement correspond donc à un écoulement dans un canal. Un profil parabolique est imposé en entrée. Les parois supérieures et inférieures et la sortie du domaine fluide sont considérées mobiles. Les efforts fluide sont calculés sur les parois supérieures et inférieures du domaine fluide et sont envoyés à la structure. La structure est modélisée par sa fibre moyenne via des éléments poutres à section circulaire et est encastrée à une extrémité. Les forces fluides des parois supérieures et inférieures sont donc confondues sur le maillage structure. Après la mise en régime de l'écoulement sur la structure au repos, alignée sur l'axe $x_{1}$, une vitesse initiale selon $x_{2}$ est donnée à la structure afin de dissymétriser l'écoulement. La fonction utilisée a la forme de la déformée modale du $2^{\mathrm{e}}$ mode propre de la structure en air afin d'accélerer le départ en instabilité qui est dû à ce mode :

$$
\begin{aligned}
v_{\mathrm{s}, 1}=A(\cos & \left(\frac{k_{2} x_{1}}{L}\right)-\cosh \left(\frac{k_{2} x_{1}}{L}\right) \\
& \left.+R_{2}\left(\sin \left(\frac{k_{2} x_{1}}{L}\right)-\sinh \left(\frac{k_{2} x_{1}}{L}\right)\right)\right)
\end{aligned}
$$

avec

$$
\left\{\begin{array}{l}
k_{2} \text { la } 2^{\mathrm{e}} \text { solution positive de } \cos (k) \cosh (k)=-1 \\
R_{2}=\frac{\sin \left(k_{2}\right)-\sinh \left(k_{2}\right)}{\cos \left(k_{2}\right)-\cosh \left(k_{2}\right)}
\end{array}\right.
$$




\begin{tabular}{|lllll|}
\hline Longueur du tube & $L$ & $=1$ & $(\mathrm{~m})$, \\
Rayon interne du tube & $R_{\mathrm{i}}=2 \times 10^{-2}$ & $(\mathrm{~m})$, \\
Épaisseur du tube & $e=4 \times 10^{-4}$ & $(\mathrm{~m})$, \\
Module d'Young & $E_{\mathrm{s}}=1,5 \times 10^{9}$ & $(\mathrm{~Pa})$, \\
Coefficient de Poisson & $\nu_{\mathrm{s}}=0,3$ & $(-)$, \\
Masse volumique de la structure & $\rho_{\mathrm{s}}=3,2 \times 10^{6}$ & $\left(\mathrm{~kg} \cdot \mathrm{m}^{-3}\right)$, \\
Masse volumique du fluide & $\rho_{\mathrm{f}}=1 \times 10^{3}$ & $\left(\mathrm{~kg} \cdot \mathrm{m}^{-3}\right)$, \\
Viscosité dynamique & $\mu$ & $=5 \times 10^{-2}$ & $\left(\mathrm{~kg} \cdot \mathrm{m}^{-1} \cdot \mathrm{s}^{-1}\right)$, \\
Vitesse de fluide en entrée & $v_{\mathrm{f}}=\left(0,614 \bar{v}_{\mathrm{red}} ; 0 ; 0\right)$ & $\left(\mathrm{m} \cdot \mathrm{s}^{-1}\right)$, \\
Vitesse réduite adimensionnée & $\bar{v}_{\mathrm{red}} \in[0,7]$ & $(-)$, \\
Amplitude initiale de la structure & $A$ & $=1,84 \times 10^{-6}$ & $\left(\mathrm{~m} \cdot \mathrm{s}^{-1}\right)$. \\
\hline
\end{tabular}

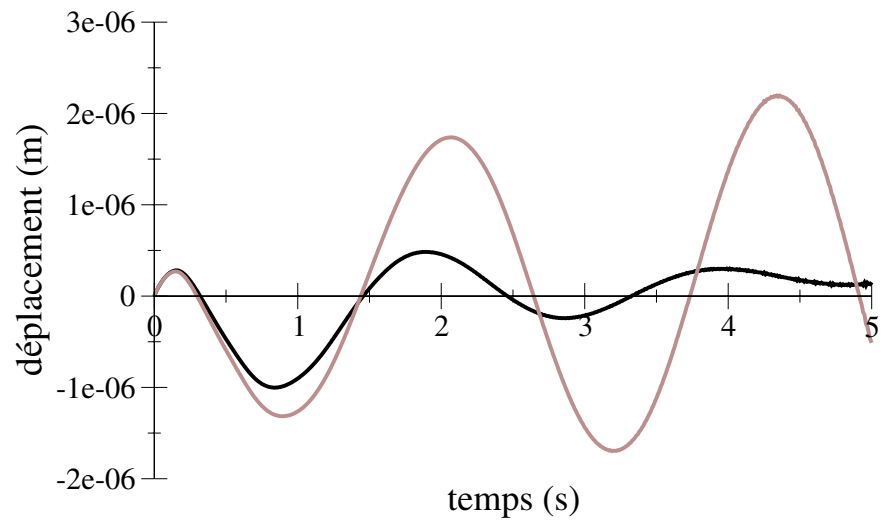

- vitesse réduite 4.0 - vitesse réduite 4.5

Fig. 4. Déplacement de l'extrémité libre de la structure pour des vitesses réduites de 4,0 et 4,5 .

Un schéma de couplage par sous-itération est utilisé afin d'assurer la convergence des interfaces fluide et structure à chaque itération. La simulation est réalisée afin d'obtenir plusieurs périodes d'oscillations pour permettre le post-traitement et l'obtention de la fréquence et de l'amortissement du deuxième mode propre en eau de la structure. La configuration choisie est celle traitée dans [20] :

$$
\text { voir le tableau ci-dessus. }
$$

\subsection{Résultats}

Des simulations ont été réalisées pour des vitesses réduites allant de 1 à 7 . Le tracé du déplacement de l'extrémité libre de la structure pour des vitesses réduites de 4 et 4,5 (Fig. 4) montre que dans le cas où $\bar{v}_{\text {red }}=4$ la structure revient vers une position d'équilibre, tandis que pour $\bar{v}_{\text {red }}=4,5$, le mouvement de la structure s'amplifie au cours du temps. Le départ en instabilité est donc compris entre ces deux valeurs. La figure 5 montre que la méthode ALE et la méthode de transpiration captent le départ en instabilité (annulation de l'amortissement)

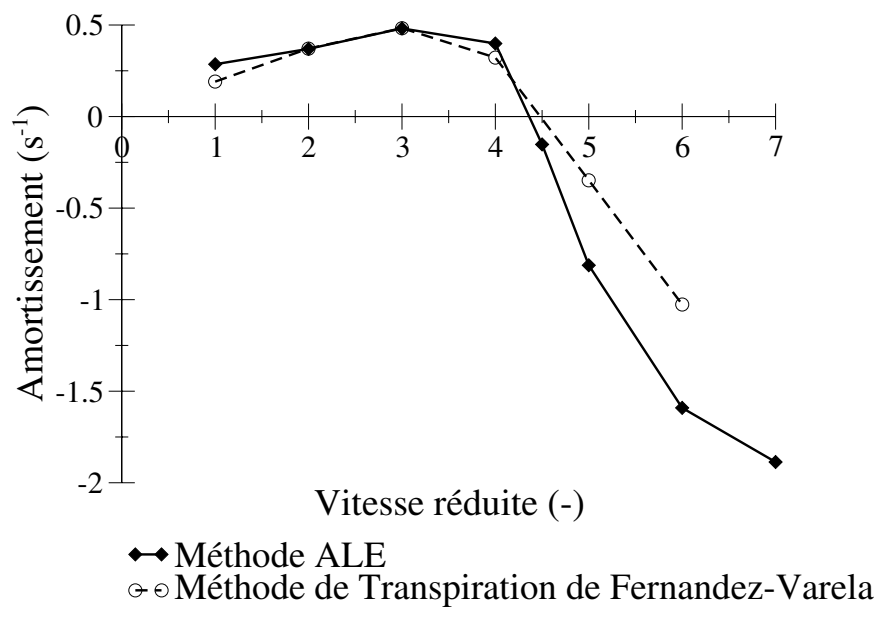

Fig. 5. Tracé de l'amortissement pour différentes vitesses réduites en entrée du fluide.

dans une même plage de vitesses réduites. Une comparaison plus précise des fréquences et des amortissements fait apparaitre une différence entre les modèles. Tant que la structure reste stable, les amortissements calculés sont du même ordre (Fig. 5). Pour des vitesses supérieures à la vitesse critique les différences s'accentuent entre les deux méthodes. À l'inverse, le calcul de la fréquence (Fig. 6) pour la méthode ALE et la méthode par transpiration de [20] sont semblables pour des valeurs éloignées de la vitesse réduite de départ en instabilité et diffèrent dans la zone de départ en instabilité.

\section{Conclusions}

Un outil de couplage externe de codes (Cosmethyc), appliqué à des structures flexibles, a été développé pour assurer la communication entre un code de mécanique des fluides (Code_Saturne) et un code de mécanique des structures (Code_Aster). L'utilisation de cette chaine de calcul au cas d'un tube soumis à un écoulement interne a permis de valider la méthodologie sur des configurations de petits déplacements et des écoulements laminaires. Les comportements stable et instable de la structure ont pu être mis en évidence. Une extension, d'une part, à des grands 


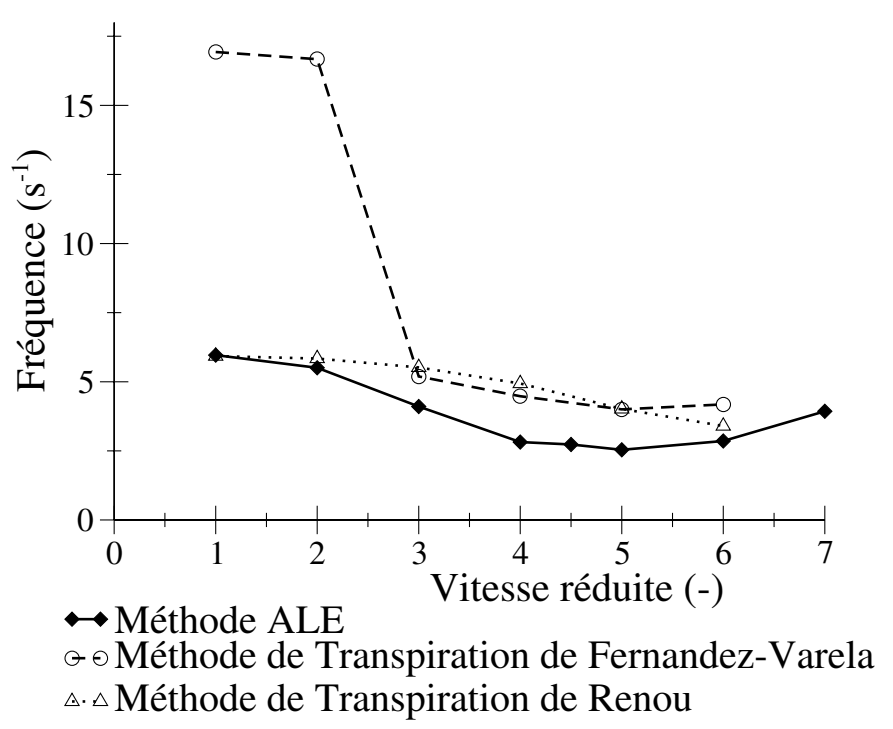

Fig. 6. Tracé de la fréquence de la structure pour différentes vitesses réduites en entrée du fluide.

déplacements structure, faisant intervenir des modèles mécaniques non-linéaires et des méthodes ALE de grandes déformations de maillage, et, d'autre part, à l'intégration de modèles de turbulences à des méthodes ALE sont envisagées pour la suite de l'élaboration de l'outil de couplage.

\section{Références}

[1] J. Blom, A monolithical fluid-structure interaction algorithm applied to the piston problem, Computer methods in applied mechanics and engineering 167 (1998) 369-391

[2] S. Etienne, D. Pelletier, A general approach to sensitivity analysis of fluid-structure interactions, J. Fluids Structures 21 (2005) 169-186

[3] B. Hübner, E. Walhorn, D. Dinkler, A monolithic approach to fluid-structure interaction using space-time finite elements, Computer methods in applied mechanics and engineering 193 (2004) 2087-2104

[4] S. Piperno, Explicit/implicit fluid/structure staggered procedures with a structural predictor and fluid subcycling for $2 \mathrm{~d}$ inviscid aeroelastic simulations, Int. J. Numerical Methods in Fluids 25 (1997) 1207-1226

[5] C. Farhat, M. Lesoinne, P. Stern, S. Lantéri, High performance solution of three-dimensional nonlinear aeroelastic problems via parallel partitioned algorithms: methodology and preliminary results, Adv. Eng. Software 28 (1997) 43-61

[6] D. Abouri, A. Parry, A. Hamdouni, Fluid-rigid body dynamic interaction in complex industrial flow, Adv. fluid Mechanics: Fluid structure interaction II, Wit Press (2003) 295-305
[7] A. de Boer, A.H. van Zuijlen, H. Bijl, Review of coupling methods for non-matching meshes, Computer methods in applied mechanics and engineering, 2006 doi : 10.1016/j.cma.2006.03.01

[8] C. Farhat, M. Lesoinne, P. LeTallec, Load and motion transfer algorithms for fluid/structure interaction problems with non-matching discrete interfaces : Momentum and energy conservation, optimal discretization and application to aeroelasticity, Computer methods in applied mechanics and engineering 157 (1998) 95-114

[9] N. Maman, C. Farhat, Matching fluid and structure meshes for aeroelastic computations a parallel approach, Computers \& Structures 54-4 (1995) 779-785

[10] J. Donea, A. Huerta, J.-Ph. Ponthot, A. RodriguezFerran, Encyclopedia of Computational Mechanics, Vol. 1: Fundamentals, chapter Chapter 14: Arbitrary Lagrangian-Eulerian Methods, John Wiley \& Sons, Ltd, 2004

[11] M. Souli, J.P. Zolesio, ALE formulation for fluid-structure interaction problems, Computer methods in applied mechanics and engineering 191 (2000) 451-466

[12] V. Guimet, Analyse numérique et simulation de problèmes d'intéraction fluide-structure en régime incompressible, thèse de doctorat, Université Paris VI, 1998

[13] L. Longatte, V. Verreman, Z. Bendjeddou, M. Souli, Comparison of strong and partitioned fluid structure code coupling methods, in Proceeding of Insert Conference Abbreviation: 2005 ASME Pressure Vessels \& Piping, Division Conference, Denver, Colorado, USA, July 1721, 2005

[14] C. Farhat, M. Lesoinne, N. Maman, Mixed explicit/implicit time integration of coupled aeroelastic problems: three field formulation, geometric conservation and distribution solution, Int. J. Numerical Methods in Fluids 21 (1995) 807-835

[15] S. Piperno, C. Farhat, B. Larrouturou, Partitioned procedures for the transient solution of coupled aeroelastic problems - part I: Model problem, theory and twodimensional application, Computer methods in applied mechanics and engineering 124 (1995) 79-112

[16] P.D. Thomas, C.K. Lombard, Geometric conservation law and its application to flow computations on moving grids, AIAA J. 17 (1979) 1030-1037

[17] M.P. Païdoussis, Dynamics of flexible slender cylinders in axial flox, Part 1, theory, J. Fluid Mech. 26-4 (1966) $717-736$

[18] J.L. Lopes, M.P. Païdoussis, C. Semler, Linear and nonlinear dynamics of cantilevered cylinders in axial flow, Part 2: the equations of motion, J. Fluids and Structures, 16-6 (2002) 715-737

[19] M.A. Fernandez Varela, Modèles simplifiés d'intéraction fluide-structure, thèse de doctorat, Université Paris IX Dauphine, 2001

[20] J.Y. Renou, Une méthode eulérienne pour le calcul numérique de forces fluides-élastiques, thèse de doctorat, Université Paris VI, 1998 